\title{
Disrupción de la educación remota en el programa universitario para adultos en tiempos de covid-19
}

\author{
Disruption of remote education in the university program for \\ adults in times of covid-19
}

\author{
Victor Fabian Romero-Escalante ${ }^{1 \mathrm{a}}$ \\ Universidad César Vallejo $^{1}$
}

(D) ORCID: https://orcid.org/0000-0002-7186-9411 ${ }^{1}$

Recibido: 12 de setiembre de 2020

Aceptado: 17 de noviembre de 2020

\section{Resumen}

La presente investigación tuvo como objetivo comparar las percepciones que tiene los alumnos del programa de formación para adultos de la facultad de administración de la Universidad Cesar Vallejo sobre la disrupción de la modalidad remota en la educación presencial. El enfoque de la investigación fue cuantitativo, el diseño cuasiexperimental y el nivel comparativo-causal expostfacto. La muestra fue conformada por 25 alumnos a quienes se le aplicó el cuestionario de percepciones de Zoller (1992), instrumento que demostró validez y confiabilidad. Los resultados indican que existe diferencias significativas entre los grupos ( $\mathrm{t}=4,67$; sig. $=0.000<0.05)$, la media más alta fue la modalidad presencial, concluyendo que los alumnos del programa de formación para adultos tienen resistencia al cambio de modalidad en la enseñanza.

Palabras claves: aprendizaje, educación comparada, educación de adultos, enseñanza a distancia, educación permanente.

\begin{abstract}

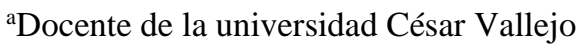

E-mail: romerofabian32@gmail.com
\end{abstract}

The objective of this research was to compare the perceptions that students of the adult training program of the Cesar Vallejo University School of Administration have on the disruption of remote modality in classroom education. The research focus was quantitative, with a quasi-experimental design and an ex-facto comparative-causal level. The sample was made up of 25 students to whom the Zoller (1992) perceptions questionnaire was applied, an instrument that demonstrated validity 
and reliability. The results indicate that there are significant differences between the groups $(\mathrm{t}=$ 4.67 ; sig. $=0.000<0.05$ ), the highest mean was the classroom education modality, concluding that the students of the adult training program have resistance to changing modality in education.

Keywords: Learning, comparative education, adult education, distance study, lifelong learning.

\section{Introducción}

A finales del año 2019 se detectó en la ciudad de Wuhan (China) un tipo de neumonía de causa desconocida, tras los alarmantes niveles de contagios y después de varias misiones realizadas por parte de la Organización Mundial de la Salud, el 11 de marzo se declara al COVID-19 como una pandemia (OMS, 2020; Adhanom, 2020). Ante ello, las universidades de China fueron las primeras en cambiar la educación presencial por una educación virtual (Abreu, 2020). En Europa, el mayor impacto social y educativo se dio principalmente en Italia y España, se cerraron todo tipo de actividades universitarias, manteniendo solo las modalidades a distancia y "on-line", por ello, los docentes debieron improvisar con los medios tecnológicos que tenían a disposición, incluso utilizando sus propias laptops y móviles (Sepie, 2020; BOE, 2020; Díez y Gajardo, 2020).

En América latina y el Caribe, las plataformas y sistemas de gestión de aprendizaje necesarios para poder construir modelos de emergencia para una educación remota no estuvieron desarrollados o están en fase preliminar de implementación (BID, 2020), si la modalidad presencial no garantiza una educación universitaria de calidad, el contexto de una educación remota es un reto muy desesperanzador, son varios los países de América Latina que han sido identificados por sus altos niveles de desigualdad en ingresos y educación, y esto conlleva a que no se pueda garantizar el acceso a educación inclusiva y de calidad (Quintana, 2020). Se calculó que unos 1,400 millones de estudiantes fueron afectados por el cierre de sus centros educativos debido al COVID-19 (UNESCO, 2020). Por lo tanto, para poder garantizar la continuidad del aprendizaje se realizó el uso intensivo de todos las plataformas y los recursos tecnológicos de manera no planificada (IESALC y UNESCO, 2020). Todo ello generó grandes dificultades incrementándose los procesos de exclusión y marginación (Ordorika, 2020).

En el Perú, el 6 de marzo se oficializa el primer caso de COVID-19 (El Comercio, 2020), en los días siguientes se suspendieron los servicios educativos tanto públicas como privadas por emergencia nacional sanitaria y el Ministerio de educación como máxima autoridad quedó autorizado para orientar sobre los mecanismos remotos que serán utilizados en todas las 
modalidades sujetos a una futura fiscalización (Decreto Supremo No 008-2020-SA, 2020; Decreto de Urgencia No 026-2020, 2020; Resolución Viceministerial Nº84-2020-MINEDU, 2020; Ley $\mathrm{N}^{\mathrm{o}} 30220$, 2014), es por ello que las universidades realizaron los ajustes necesarios para implementar una educación remota con las herramientas tecnológicas disponibles de cada casa de estudio.

En lo que respecta a la educación universitaria para adultos, es aquella que se le brinda mediante horarios flexibles y técnicas andragógicas de enseñanza-aprendizaje, al sector de la población que por motivos generacionales, laborales y familiares no pueden optar por los horarios regulares que ofrecen las universidades (Ramírez y Victor-Ramírez, 2010), este tipo de educación surgió en la década de los ochenta en las universidades de varios países desarrollados cumpliendo una importante función cultural y socializadora (Valle, 2014). Las necesidades educativas que tienen las personas mayores según McClusky (1982), citado por Valle (2014), se agrupan en cinco dimensiones: necesidades de adaptarse al proceso de envejecimiento, necesidades de creatividad, necesidades de información, necesidades relacionados al significado de la vida y necesidades de tener información para influenciar en la vida social. Lo mencionado difiere de las motivaciones que tienen alumnos universitarios en edad adolescente para elegir una carrera universitaria, las mismas que oscilan entre el empleo, el prestigio y el poder (Rodríguez-Muñiz, Areces, SuárezAlvarez, Cueli y Muñiz, 2019).

En cuanto a las modalidades de educación, la presencial, llamada también enseñanza tradicional, es la que se brinda en persona sin requerimiento de contenidos virtuales, la enseñanza se da a través de su comportamiento no verbal y verbal para desarrollar los aprendizajes cognitivos y afectivos en los estudiantes. Es el pilar de la enseñanza superior en muchas de las universidades y base de la propagación de conocimientos durante siglos (Martínez, 2017; Bachelor, 2019; Jardines, 2010). Esta modalidad estaba migrando muy lentamente a la educación virtual, antes de la pandemia tenía un crecimiento mundial del 3\% (Abreu, 2020). Por otro lado, la educación remota universitaria, a diferencia de la educación virtual o semipresencial propiamente dicho, es aquel aprendizaje que sufrió la transformación de presencial a una virtual después de la declaración de la pandemia mundial (MINEDU, 2020), lo que hace es trasladar la educación presencial a las plataformas virtuales, generando una disrupción pedagógica, con tecnologías aplicadas a la innovación educativa que poco a poco desplazaran la educación tradicional y abriendo camino a una educación diseñada para las nuevas generaciones (Abreu, 2020; García, 2017; Juca, 2016). 
A la luz de todo lo considerado, el objetivo de la investigación se dirige a comparar las percepciones que tienen los alumnos del programa de formación para adultos de la facultad de administración de la Universidad Cesar Vallejo (Lima, Perú) sobre la disrupción de la modalidad remota en la educación presencial.

\section{Metodología}

La presente investigación tiene un enfoque cuantitativo debido a que se utilizó un cuestionario para la recolección de datos y mediante el análisis estadístico se determinó el comportamiento de la variable en estudio (Arias, 2012); el diseño es cuasiexperimental, puesto que observaremos el comportamiento de la variable sobre la misma muestra que ya están formados previamente, con una medición previa y posterior, sin contar con un grupo de control (Ramírez, 2016; Silvestre y Huamán, 2019), asimismo, el estudio fue comparativo-causal expost-facto porque se propuso establecer comparaciones entre dos grupos de muestras para establecer diferencias (Bisquerra, 2009).

Participaron del estudio 25 alumnos de la Facultad de Administración del programa de formación para adultos que en el semestre 2019-2 llevaron el curso de Proyecto de Investigación de manera presencial y en el semestre 2020-1 llevaron el curso de Desarrollo de Proyecto de Investigación totalmente de manera remota, siendo la característica principal del estudio que son los mismos alumnos y el mismo docente, por lo tanto, lo que se pretende evaluar es la percepción que tienen los alumnos sobre la enseñanza impartida ante el cambio de contexto por la emergencia.

La técnica utilizada fue la encuesta y el instrumento utilizado fue la adaptación del cuestionario originalmente elaborado por Zoller (1992), asimismo utilizado por diversos autores en sus investigaciones para medir percepciones de los alumnos sobre un determinado curso a partir de sus propias experiencias (Cho y Baek, 2019; López, Alarcón, Rodríguez y Casado, 2014; Chiecher, Donolo y Rinaudo, 2010, Paoloni, 2009; Chiecher, Donolo y Rinaudo, 2005), el mismo contiene 17 preguntas que abarcan 4 dimensiones: (i) características de las clases (7 ítems); (ii) Diseño del curso (5 ítems); (iii) Desarrollo de la autonomía (3 ítems) y (iv) calidad de la enseñanza (2 ítems); los ítems del cuestionario están sobre una base de la escala de Likert de 6 puntos (muy mala, mala, regular, buena, muy buena, excelente), el instrumento se aplicó una vez culminado cada semestre de estudio. La validación se realizó a través de juicio experto con un coeficiente $\mathrm{V}$ 
de Aiken de 0,85, asimismo tras realizar un piloto del cuestionario se obtuvo un coeficiente de alfa de Cronbach de 0,946, siendo el cuestionario confiable y valido para realizar el estudio comparado.

Para el análisis estadístico se utilizó el software SPSS versión 24 con la cual se realizaron las pruebas T de Student para muestras relacionadas en las variables tienen una distribución normal y la prueba no paramétrica de Wilcoxon para las dimensiones que no cumplieron la distribución normal.

\section{Resultados}

Según el tratamiento estadístico de los datos se observa diferencias significativas entre los grupos semestre 2019-2 del curso de proyecto de investigación bajo modalidad presencial y semestre 2020-1 del curso desarrollo de proyecto de investigación bajo la modalidad remota. La tabla 1 nos muestra los resultados de las medias y desviaciones estándar de los dos grupos observados en cada dimensión considerada.

Tabla 1

Media y desviación estándar de la percepción del aprendizaje de los alumnos

\begin{tabular}{|c|c|c|c|c|}
\hline \multirow{2}{*}{$\begin{array}{c}\text { Ítems } \\
\text { Percepción global del alumno }\end{array}$} & \multicolumn{2}{|c|}{$\begin{array}{c}\text { Semestre } 2019-2 \text { modalidad } \\
\text { presencial }\end{array}$} & \multicolumn{2}{|c|}{$\begin{array}{l}\text { Semestre 2020-1 } \\
\text { modalidad remota }\end{array}$} \\
\hline & $\overline{\mathrm{X}}=89,20$ & $\mathrm{Sd}=8,59$ & $\overline{\mathrm{X}}=80,44$ & $\mathrm{Sd}=9,87$ \\
\hline Características de la clase & $\bar{X}=38,80$ & $\mathrm{Sd}=2,04$ & $\bar{x}=32,92$ & $\mathrm{Sd}=4,49$ \\
\hline Diseño del curso & $\bar{X}=25,40$ & $\mathrm{Sd}=3,97$ & $\bar{X}=23,12$ & $\mathrm{Sd}=3,21$ \\
\hline Desarrollo de la autonomía & $\bar{X}=14,92$ & $\mathrm{Sd}=2,38$ & $\bar{X}=14,44$ & $\mathrm{Sd}=2,10$ \\
\hline Calidad de la enseñanza & $\overline{\mathrm{X}}=10,08$ & $\mathrm{Sd}=1,55$ & $\bar{x}=9,96$ & $\mathrm{Sd}=1,46$ \\
\hline
\end{tabular}

Nota: $\overline{\mathrm{X}}=$ media; $\mathrm{Sd}=$ desviación estándar

Mediante la prueba de Shapiro-Wilk, por tener un grado de libertad menor a 50, se verificó los supuestos de normalidad de la distribución de los datos que integran las diferencias de las medias tomadas antes de manera presencial (pretest) y después de manera remota (post test), los resultados de esta prueba se encuentran en la Tabla 2. Según esta información se realizó la prueba t para muestras relacionadas sobre la percepción global del aprendizaje de los alumnos y las dimensiones diseño del curso y calidad de la enseñanza, debido a que los puntajes obtenidos registran una distribución normal. En cuanto a las dimensiones características de clase y desarrollo 
de la autonomía se halló evidencias para rechazar la hipótesis nula de normalidad por lo tanto se tuvo que aplicar la prueba no paramétrica de Wilcoxon.

Tabla 2

Prueba de normalidad de Shapiro-Wilk

\begin{tabular}{lccc} 
& Estadístico & Grado de libertad & Sig. \\
\hline Diferencia en la Percepción general &, 939 & 25 &, 138 \\
Diferencia en las Características de clase &, 898 & 25 &, 017 \\
Diferencia en el Diseño del curso &, 951 & 25 &, 263 \\
Diferencia en el Desarrollo de la autonomía &, 806 & 25 &, 000 \\
Diferencia en la Calidad de la enseñanza &, 921 & 25 &, 053 \\
\hline
\end{tabular}

En cuanto a los resultados de las pruebas de muestras relacionadas mostradas en la tabla 3 y tabla 4, tenemos que la percepción global de los alumnos sobre el aprendizaje se observaron diferencias estadísticas significativas $(\mathrm{t}=4,67$; $\mathrm{sig}=.000)$ entre semestre 2019-2 del curso de proyecto de investigación bajo modalidad presencial $(\overline{\mathrm{X}}=89,20 ; \mathrm{Sd}=8,59)$ y semestre 2020-1 del curso desarrollo de proyecto de investigación bajo la modalidad remota $(\overline{\mathrm{x}}=80,44 ; \mathrm{Sd}=9,87)$. Con respecto a la dimensión características de la clase se observaron diferencias estadísticas significativas $(Z=-4,183$; sig = .000) entre semestre 2019-2 del curso de proyecto de investigación bajo modalidad presencial $(\bar{x}=38,80 ; S d=2,04)$ y semestre 2020-1 del curso desarrollo de proyecto de investigación bajo la modalidad remota $(\overline{\mathrm{x}}=32,92 ; \mathrm{Sd}=4,49)$. Por lo que se refiere a la dimensión diseño del curso se observaron diferencias estadísticas significativas $(\mathrm{t}=3,343$; $\operatorname{sig}=$ .003) entre semestre 2019-2 del curso de proyecto de investigación bajo modalidad presencial $(\overline{\mathrm{x}}=$ 25,40; Sd = 3,97) y semestre 2020-1 del curso desarrollo de proyecto de investigación bajo la modalidad remota $(\bar{x}=23,12 ; S d=3,21)$. Sobre las dimensiones desarrollo de la autonomía $(Z=$ ,000; sig = .316) y calidad de la enseñanza $(\mathrm{t}=, 391$; sig $=.700)$, no se hallaron diferencias estadísticas significativas por lo que se infiere que la percepción de los alumnos es similar en ambas modalidades.

Tabla 3

Prueba t de Student de muestras relacionadas 


$\begin{array}{ccccccccc} & \text { Media } & \begin{array}{c}\text { Desviación } \\ \text { estándar }\end{array} & \begin{array}{c}\text { Media } \\ \text { de error } \\ \text { estándar }\end{array} & \begin{array}{c}\text { 95\% de IC de la } \\ \text { diferencia }\end{array} & \text { therior } & \text { superior } & \text { gl } & \text { sig } \\ \text { (bilateral) }\end{array}$

Tabla 4

Prueba de rangos con signo de Wilcoxon para muestras relacionadas

\begin{tabular}{ccccccc} 
& N & $\begin{array}{c}\text { Rango } \\
\text { promedio }\end{array}$ & $\begin{array}{c}\text { Suma de } \\
\text { rangos }\end{array}$ & Z & $\begin{array}{c}\text { Sig. Asintótica } \\
\text { (bilateral) }\end{array}$ \\
\hline $\begin{array}{c}\text { Características de las } \\
\text { clases (remota) - }\end{array}$ & Rangos negativos & 22 & 13.45 & 296.00 & & \\
Características de las & Rangos positivos & 2 & 2.00 & 4.00 & $-4,183$ &, 000 \\
clases (presencial) & Empates & 1 & & & & \\
Total & 25 & & & \\
Desarrollo de la & Rangos negativos & 8 & 8.50 & 68.00 & & \\
autonomía (remota) - & Rangos positivos & 6 & 6.17 & 37.00 & & \\
Desarrollo de la & Empates & 11 & & & & \\
autonomía (presencial) & Total & 25 & & & & \\
\hline
\end{tabular}

\section{Discusión}

El objetivo de la presente investigación fue comparar las percepciones que tiene los alumnos del programa de formación para adultos de la facultad de administración de la Universidad Cesar Vallejo sobre la disrupción de la modalidad remota en la educación presencial. Un primer hallazgo indica que los alumnos de formación adulta tuvieron una percepción más positiva en la modalidad presencial, de acuerdo con el estudio, en dos de las cuatro dimensiones (características de clase y diseño del curso) se encontraron suficientes evidencias estadísticas para inferir que los alumnos en estudio prefieren la enseñanza tradicional a la enseñanza remota de emergencia, ello 
engloba una serie factores que fueron desarrollados en doce ítem de los diecisiete, que puede asumirse como una típica resistencia al cambio, estos resultados obtenidos concuerdan con un estudio realizado en personas mayores, donde se menciona que los medios electrónicos o digitales, según la teoría andragógica, son menos efectivos en el aprendizaje cuando los participantes son adultos (Kaupins, 2002, como se citó en Jardines, 2010).

Por su parte, Martínez (2017) realizó el análisis de las ventajas y desventajas entre la educación presencial y a distancia, concluyendo rotundamente que ambas modalidades coexistirán en el futuro potenciándose mutuamente, la modalidad presencial dejaría de ser $100 \%$ presencial enriquecida por técnicas b-learnig. Sobre este último enfoque ya se viene dando en los programas de educación adulta y con mucho éxito en programas de postgrado en países desarrollados (Abreu, 2020), por ende ambas modalidades convivirán por mucho tiempo.

Con respecto a la disrupción educativa, en la producción académica todavía no se ha desarrollado estudios sobre cómo los alumnos de edad adulta perciben este cambio brusco de la modalidad de educación, las investigaciones generalmente apuntan a la irrupción de la educación virtual con los modelos tecnológicos de las cuales se soporta y con alumnos universitarios del sistema regular, por ejemplo, Chiecher, Donolo y Rinaudo (2005) quienes realizaron su investigación con la participación de 175 estudiantes, 70 de los cuales llevaron cursos presenciales durante el año 1999 y 105 alumnos cuando se implementó la modalidad mixta entre presencial y a distancia durante al año 2004, llegaron a la conclusión que la educación mixta tuvo mayor impacto en las experiencias de los estudiantes. Los mismos autores, Chiecher, Donolo y Rinaudo (2010), repitieron la observación con 50 alumnos del último año de la licenciatura en psicopedagogía mayoritariamente mujeres (94\%) pero en esta ocasión el mismo grupo fue observado en actividades presenciales y aulas virtuales, concluyendo que las experiencias más positivas de los alumnos fueron en el aula virtual.

De igual manera, Fernández-Pascual, Ferrer-Cascales y Reig-Cascales (2013) en su investigación para hallar el grado de satisfacción de los estudiantes en cuanto al proceso de enseñanza-aprendizaje en un ámbito semipresencial, aplicando una adaptación del cuestionario The Distance Education Learning Environments Survey (Sp-DELES), a 265 universitarios con una edad media de 30.7 años, llegando a la conclusión que la mayoría de los estudiantes se encuentran muy satisfechos con la aplicación de entornos virtuales como metodología de enseñanzaaprendizaje. Por otro lado, Jardines (2010) hizo una comparación entre la educación a distancia 
con la educación presencial y en lo referente al rendimiento académico de los estudiantes, no encontró diferencias significativas.

Finalmente, se precisa que el presente estudio tuvo algunas limitantes, como el tamaño de la muestra, que al ser un curso taller de proyecto y desarrollo de tesis, impartida en la carrera de administración de la universidad César Vallejo no se podría generalizar los hallazgos a todas las carreras de la universidad por las líneas de investigación diferenciadas que tienen, quedando restringida al presente grupo de participantes, por ello, sería recomendable ampliar el estudio con participantes de otras carreras y así poder generalizar los resultados. Sin embargo, estos resultados nos dan una primera aproximación de la percepción que tienen los alumnos sobre la disrupción de la modalidad remota en el programa para adultos y así poder generar estrategias que permitan un mejor servicio educativo.

\section{Conclusiones}

Los hallazgos del presente estudio permitieron verificar diferencias estadísticas significativas de la percepción global de los alumnos sobre el aprendizaje entre modalidad presencial y remota, la media más alta fue obtenida bajo la modalidad presencial, estos resultados nos indican que los alumnos han obtenido una mejor experiencia cuando se impartían las clases de manera directa en el aula, ello también puede darse por la resistencia al cambio que experimentan los alumnos sobre las nuevas tecnologías virtuales, debido a que están en un programa de educación andragógica la cuál es exclusiva para adultos.

Por otro lado, se observó que, de las 4 dimensiones estudiadas de la percepción sobre el aprendizaje de acuerdo con la experiencia de los alumnos, se encontraron diferencias significativas en características de la clase y diseño del curso, por lo revisado en los ítems se observa que los alumnos de formación adulta prefieren la educación impartida de manera tradicional con el profesor en clase, los medios habituales del salón de clases y las calificaciones de manera presencial. Se recomienda que futuros estudios amplíen la gama de la muestra con otras escuelas para poder generalizar los resultados, ampliando a una investigación mixta para obtener una mayor información sobre la resistencia a un cambio del paradigma educativo; a la vez que debe generarse una sincronización y empatía entre los docentes, los alumnos y la universidad, para así, poder eliminar las brechas que se tienen en cuanto al uso de las tecnologías de información. 


\section{Referencias}

Abreu, J. (2020). Tiempos de Coronavirus: La Educación en Línea como Respuesta a la Crisis. Revista Daena (International Journal of Good Conscience), 15(1), pp. 1-15. http://search.ebscohost.com/login.aspx?direct=true\&db=fua\&AN=144462776\&lang=es\& $\underline{\text { site }=\text { eds-live }}$

Adhanom, T. (2020, 11 de marzo). Alocución de apertura del Director General de la OMS en la rueda de prensa sobre COVID-19 celebrada el 11 de marzo del 2020. [Web oficial de la Organización Mundial de la Salud]. Recuperado de: https://www.who.int/es/dg/speeches/detail/who-director-general-s-opening-remarks-atthe-media-briefing-on-covid-19---11-march-2020

Arias, F. (2012). El proyecto de investigación. Introducción a la metodología científica (Sexta Edición). Caracas: Editorial Episteme

Bachelor, J. (2019). El aula presencial, semipresencial, virtual e invertida: Un estudio comparativo de métodos didácticos en la enseñanza de L2. Revista Educación, 43(2). https://doi.org/10.15517/revedu.v43i2.34014

BID. (2020, mayo). La educación en tiempos del coronavirus. Los sistemas educativos de América $\begin{array}{llll}\text { Latina } & \text { el Caribe ante COVID-19. }\end{array}$ https://publications.iadb.org/publications/spanish/document/La-educacion-en-tiemposdel-coronavirus-Los-sistemas-educativos-de-America-Latina-y-el-Caribe-ante-COVID19.pdf

Bisquerra, R. (2009). Metodología de la investigación educativa (Segunda Edición). Madrid: Editorial La Muralla.

BOE. (2020, 14 de marzo). Real Decreto 463/2020, de 14 de marzo, por el que se declara el estado de alarma para la gestión de la situación de crisis sanitaria ocasionada por el COVID-19. Boletín Oficial del Estado Español. Recuperado de: https://www.boe.es/eli/es/rd/2020/03/14/463/con

Chiecher, A., Donolo, D. y Rinaudo, M. (2010). Tomando opciones en la universidad: entre el aprendizaje presencial y a distancia. Innovación educativa. 10(52), pp. 45-53. https://www.redalyc.org/pdf/1794/179420763005.pdf 
Chiecher, A., Donolo, D. y Rinaudo, M. (2005). Percepciones del aprendizaje en contextos presenciales y virtuales. La perspectiva de alumnos universitarios. Revista de Educación a Distancia (RED). (13). https://revistas.um.es/red/article/view/24401

Cho, J. y Baek, W. (2019). Identifying Factors Affecting the Quality of Teaching in Basic Science Education: Physics, Biological Sciences, Mathematics, and Chemistry. Sustainability, 11(14). https://doi.org/10.3390/su11143958

Decreto de Urgencia No 026-2020 (2020, 15 de marzo). Decreto de urgencia que establece diversas medidas excepcionales y temporales para prevenir la propagación del coronavirus (covid19) en el territorio nacional. Normas Legales. Diario Oficial El Peruano, pp. 1-10. https://www.gob.pe/institucion/presidencia/normas-legales/460471-026-2020

Decreto Supremo No 008-2020-SA (2020, 11 de marzo). Decreto Supremo que declara en Emergencia Sanitaria a nivel nacional por el plazo de noventa (90) días calendario y dicta medidas de prevención y control del COVID-19. Normas Legales. Diario Oficial El Peruano, pp. 6-7. https://www.gob.pe/institucion/minsa/normas-legales/483010-008$\underline{2020-s a}$

Díez, E. \& Gajardo, K. (2020). Educating and Evaluating in Times of Coronavirus: the Situation in Spain. REMIE - Multidisciplinary Journal of Educational Research. 10(2), pp. 102-134. https://doi.org/10.17583/remie.2020.5604

El Comercio. (2020, 7 de marzo). Primer contagiado es un aviador comercial. El coronavirus en el Perú [Edición Especial]. El comercio, Año 180, № 90,719, p. A4 [Edición impresa]

Fernández-Pascual, M.; Ferrer-Cascales, R. y Reig-Ferrer, A. (2013). Entornos virtuales: predicción de la satisfacción en contexto universitario. Píxel-Bit. Revista de Medios y Educación. pp. 167-181. https://recyt.fecyt.es/index.php/pixel/article/download/61556/37569/0

García, L. (2017). Educación a distancia y virtual: calidad, disrupción, aprendizajes adaptativo y móvil. RIED. Revista Iberoamericana de Educación a Distancia, 20(2), pp. 9-25. http://www.redalyc.org/articulo.oa?id=331453132001

IESALC y UNESCO (2020, 13 de mayo). COVID-19 y educación superior: De los efectos inmediatos al día después. Análisis de impactos, respuestas políticas y recomendaciones. http://www.iesalc.unesco.org/wp-content/uploads/2020/05/COVID-19-ES-130520.pdf 
Jardines, F. (2010). La educación a distancia: Una comparación con la educación presencial, en relación con la función del profesor, del estudiante y de los medios. Innovaciones de Negocios, 7(1), pp. 177 -190. http://eprints.uanl.mx/12535/1/A9.pdf

Juca, F. (2016). La educación a distancia, una necesidad para la formación de los profesionales. Revista Universidad y Sociedad, 8(1). pp.106-111. http://rus.ucf.edu.cu/

Ley No 30220 (2014, 9 de julio). Ley Universitaria. Normas Legales. Diario Oficial El Peruano, Año 31, No 12914. https://www.sunedu.gob.pe/wp-content/uploads/2017/04/Leyuniversitaria-30220.pdf

López, D.; Alarcón, P.; Rodríguez, M. y Casado, M. (2014). Motivación en estudiantes de ingeniería: Un caso de estudio con teorías e instrumentos para su medida y desarrollo. REDU - Revista de Docencia Universitaria, 12(4), pp. 343-376. https://dialnet.unirioja.es/descarga/articulo/4908189.pdf

Martínez, V. (2017). Educación presencial versus educación a distancia. La Cuestión Universitaria, $\quad 9, \quad$ pp. 108-116. http://polired.upm.es/index.php/lacuestionuniversitaria/article/download/3582/3662

MINEDU (2020). Orientaciones para la continuidad del servicio educativo superior universitario, en el marco de la emergencia sanitaria por el covid-19. Resolución Viceministerial № 0852020-MINEDU. $\quad$ http://www.minedu.gob.pe/reforma-universitaria/pdf/orientacionesuniversidades.pdf

OMS. (2020, 5 de enero). Neumonía de causa desconocida - China. Brotes Epidémicos. Sección: Preparación y respuesta ante emergencias [Web oficial de la Organización Mundial de la Salud]. $\quad$ https://www.who.int/csr/don/05-january-2020-pneumonia-of-unkown-causechina/es/

Ordorika, I. (2020). Pandemia y educación superior. Revista de la Educación Superior. 49, pp. 18. https://doi.org/10.36857/resu.2020.194.1120

Paoloni, P. (2009). Contextos favorecedores de la motivación y el aprendizaje. Una propuesta innovadora para alumnos de Ingeniería. Electronic Journal of Research in Educational Psychology, 7(3), pp. 953-984. https://www.redalyc.org/pdf/2931/293121984003.pdf

Quintana, I. (2020). Covid-19 y Cierre de Universidades ¿Preparados para una Educación a Distancia de Calidad? Revista Internacional de Educación para la Justicia Social, 9(3). https://revistas.uam.es/riejs/article/view/12232/12094 
Ramírez, R. (2016). Proyecto de investigación. Cómo se hace una tesis (Segunda Edición). Lima: Fondo editorial AMADP

Resolución Viceministerial $\mathrm{N}^{\text {o }}$ 084-2020-MINEDU (2020, 1 de abril). Disponen medidas excepcionales con relación al servicio educativo que se realiza de forma presencial, correspondiente al año lectivo 2020 brindado por los Centros de Educación TécnicoProductiva e Institutos y Escuelas de Educación Superior públicos y privados. Normas Legales. Diario Oficial El Peruano, pp. https://www.gob.pe/institucion/minedu/normas-legales/466131-084-2020-minedu

Ramírez, L. y Víctor-Ramírez, A. (2010, enero-junio). Educación para adultos en el siglo XXI: análisis del modelo de educación para la vida y el trabajo en México ¿avances o retrocesos? Tiempo de Educar. 11(21), pp. 59-78. https://www.redalyc.org/pdf/311/31116163004.pdf

Rodriguez-Muñiz, L.; Areces, D.; Suárez-Álvarez, J.; Cueli, M. y Muñiz, J. (2019). ¿Qué motivos tienen los estudiantes de Bachillerato para elegir una carrera universitaria? Revista de Psicología y Educación / Journal of Psychology and Education. 14(1), pp. 1-15. https://doi.org/10.23923/rpye2019.01.167

SEPIE. (2020, 24 de febrero). Comunicado sobre el coronavirus COVID-19. http://sepie.es/doc/comunicacion/prensa/2020/COVID_19.pdf

Silvestre, I. y Huamán, C. (2019). Pasos para elaborar la investigación y la redacción de la tesis universitaria. Lima: Editorial San Marcos.

UNESCO (2020, 26 de marzo). Coalición mundial para la educación. Mensaje de Audrey Azoulay, Directora General de la UNESCO [Video]. YouTube. https://youtu.be/LGbqjso8BlA

Valle, J. (2014). Educación permanente: los programas universitarios para mayores en España como respuesta a una nueva realidad social. Revista de la Educación Superior. 3(171), pp. 117-138. https://doi.org/10.1016/j.resu.2015.03.003

Zoller, U. (1992). Faculty teaching performance evaluation in higher science education: issues and implications. Science $\quad$ Education. 676), pp. https://doi.org/10.1002/sce.3730760607 